\title{
Dihydropyrimidine dehydrogenase (DPD) activity in gastric cancer tissue and effect of DPD inhibitory fluoropyrimidines
}

\author{
Hisashi Usuki, Ken Ishimura, Shinichi Yachida, Masanobu Hagitke, Keitchi Okano, Kunihiko Izuishi, \\ Yukihiko Karasawa, Fuminori Goda, and Hajime Maeta
}

First Department of Surgery, Kagawa Medical University, 1750-1 Ikenobe, Miki-cho, Kagawa 761-0793, Japan

\begin{abstract}
Background. Dihydropyrimidine dehydrogenase (DPD) is an enzyme that catabolizes 5-fluorouracil (5-FU). The effect of DPD inhibitory fluoropyrimidines (DIF) is presumably related to DPD activity. We studied the efficacy of DIF (tegafur + uracil [UFT], tegafur + gimeracil + osteracil [S-1 (TS-1 $\left.\left.{ }^{\circledR}\right)\right]$ ) relative to DPD activity, with other fluoropyrimidines as controls.

Methods. The efficacy of DIF relative to DPD activity was evaluated in 58 gastric cancer patients who received postoperative administration of fluoropyrimidines, consisting of DIF in 42 patients (UFT in 23; S-1 in 19) and non-DIF in 16 patients.

Results. In patients with low DPD activity (under $40 \mathrm{U} / \mathrm{mg}$ protein), curative potential tended to be lower for DIF than for non-DIF, but the survival rate was the same for both. In patients with high DPD activity $(40 \mathrm{U} / \mathrm{mg}$ protein or more), such a tendency was not detected. In a comparison between those treated with UFT and those treated with S-1, prognosis was better in the latter group, in spite of their predominance of lower curative potentials of $B$ or $C$. In 27 patients with measurable lesions, a partial response (PR) or higher response occurred in $33 \%(5 / 15)$ of those with low DPD activity, and in $17 \%(2 / 12)$ of those with high DPD activity. In the patients with low DPD activity, non-DIF induced no change (NC) in 17\% (1/6), and progressive disease (PD) in the rest. UFT induced PD in all 5 patients, while $S-1$ induced a response rate of $44 \%(7 / 16)$, with $\mathrm{NC}$ in $25 \%(4 / 16)$. In the patients with high DPD activity, on the other hand, non-DIF $(n=3)$ and UFT $(n=3)$ induced PD in all the patients, while S-1 induced PR in 33\% (2/6) and NC or a higher response in 67\% (4/6).

Conclusion. It is recommended to use S-1 rather than UFT in patients with high DPD activity. Measurement of DPD was useful in drug selection.
\end{abstract}

Offprint requests to: $\mathrm{H}$. Usuki

Received: October 28, 2002 / Accepted: February 3, 2003
Key words Gastric cancer - Dihydropyrimidine dehydrogenase $\cdot \mathrm{S}-1$

\section{Introduction}

Recently, the actions of various enzymes involved in the pharmacokinetics of fluoropyrimidines have been attracting attention [1-6]. Dihydropyrimidine dehydrogenase (DPD) is one of these enzymes, and is known to play a key role in the catabolism of 5-fluorouracil (5-FU) [1]. Accordingly, DPD activities in a tumor could be very closely related to the antitumor effect of fluoropyrimidines. DPD inhibitory fluoropyrimidines (DIF) are drugs developed to increase the effect of antitumor drugs by inhibiting DPD activity, and include tegafur + uracil (UFT) [7], ethynyluracil [8], tegafur + gimeracil + osteracil (S-1) [9], and 6dihydroxypyrimidine + ethoxymethyl-5-fluorouracil (BOF-A2) [10,11]. Of these drugs, only UFT and S-1 have been approved in Japan and are now widely used in clinics. Gimeracil, contained in S-1, is believed to be approximately 180 times as potent as uracil in terms of DPD inhibitory activity [12], so that UFT and S-1 must be considered separately, although both are DIF preparations, and their effects are, presumably, closely related to DPD activity within the tumor. In the present study of the relationship between DPD activity in the tumor and the efficacy of DIF preparations, a comparison was made between DIF and other fluoropyrimidines (non-DIF), as well as between UFT and S-1.

\section{Patients and methods}

\section{Patients}

In 74 patients who underwent resection of gastric cancer between 1999 and 2001 in our department, DPD activity 
in the resected tumor was determined after their informed consent was obtained. Fluoropyrimidines were administered as adjuvant chemotherapy following gastrectomy in 58 of these patients. DIF were used in 42 patients (UFT in 23; S-1 in 19), and non-DIF (leucovorin +5 -FU) in 16 patients.

\section{Measurement of DPD}

The tissue specimens used for measuring DPD activity were collected from the tumor margins of the resected materials immediately after gastrectomy. The specimens were frozen in liquid nitrogen immediately after collection, and stored in a deep freezer at $-85^{\circ} \mathrm{C}$. DPD activity was measured quantitatively by an enzymelinked immunosorbent assay using a DPD monoclonal antibody [13].

\section{Evaluation}

Comparisons were made between the 42 patients treated with DIF and the 16 patients treated with nonDIF, with respect to background, curative potential (based on the Japanese classification of gastric carcinoma [14]), and survival rate. In patients treated with DIF, a similar comparison was made between those treated with UFT and those treated with S-1.

All patients examined for DPD activity were divided into high and low populations according to a median value of DPD activity of $40 \mathrm{U} / \mathrm{mg}$ protein or more and under $40 \mathrm{U} / \mathrm{mg}$ protein, respectively. The curative potential and survival rate were compared between the group treated with DIF and the non-DIF group in each population of DPD activity.

Similarly, in those treated with DIF, curative potential and survival rate were compared between the patients treated with UFT and those treated with S-1 in each population of DPD activity.

In patients with curative potential $\mathrm{C}$, the responses to chemotherapy were classified according to WHO criteria, and analyzed according to the DPD activity and factors limiting curative potential.

\section{Statistics}

Relationships between the drug administered and the patient's background were analyzed using the $\chi^{2}$ test, and a significance level of $P<0.05$ was used to test for correlation. Survival rate was calculated by the KaplanMeier method, and differences between two groups were tested by log rank analysis, with a significance level of $P<0.05$.

\section{Results}

\section{Patients' characteristics}

Table 1 lists the histological type, depth of tumor invasion, lymph node metastasis, liver and peritoneal metastases, and curative potential of surgery in the 58 patients, according to the drug administered. There were no significant differences in the patients' characteristics, except that S-1 was used more frequently in patients with positive peritoneal metastasis and curative potential C, while UFT was used more frequently in patients with curative potential A.

\section{Efficacy}

Survival

Correlation to drug administered. No difference in survival rate was observed between the 42 patients treated with DIF and the 16 treated with non-DIF, although patients with curative potential $\mathrm{C}$ were more frequent in the former group than in the latter (Fig. 1). Comparing the 23 patients treated with UFT and the 19 treated with $\mathrm{S}-1$, although those with a curative potential of $\mathrm{C}$ were significantly more frequent in the latter group, there was no difference in survival rate between the two groups (Fig. 2). No difference was detected in survival rate between the 23 patients treated with UFT and the 16 treated with non-DIF, either (Fig. 3).

Correlation to DPD activity. In the 26 patients with low DPD activity, the survival rate was the same in the 19 treated with DIF and the 7 treated with non-DIF. Although the number of cases was limited, curative potential tended to be lower in the former group than in the latter, indicating the efficacy of DPD inhibition by DIF

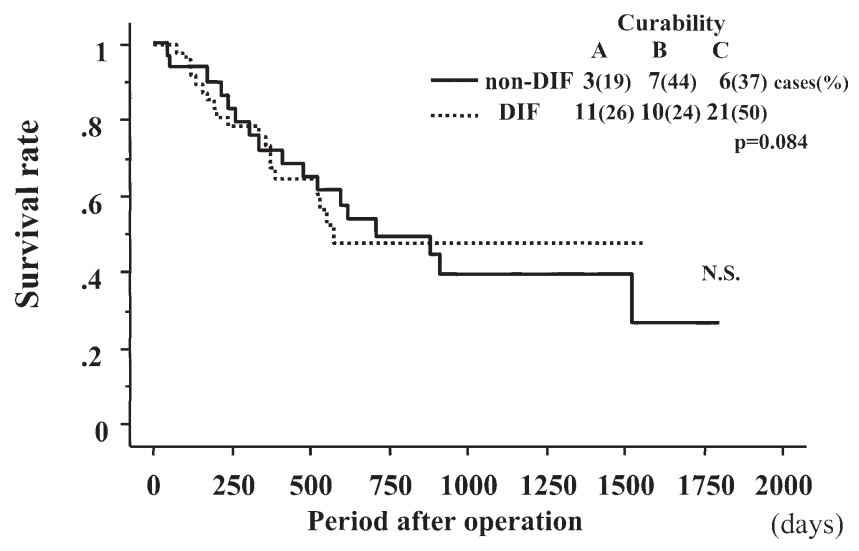

Fig. 1. Comparison of survival curves between the patients who received dihydropyrimidine dehydrogenase (DPD) inhibitory fluoropyrimidines $(D I F)$ and the patients who received other kinds of fluoropyrimidine. N.S. Not significant 
Table 1. Patients' characteristics

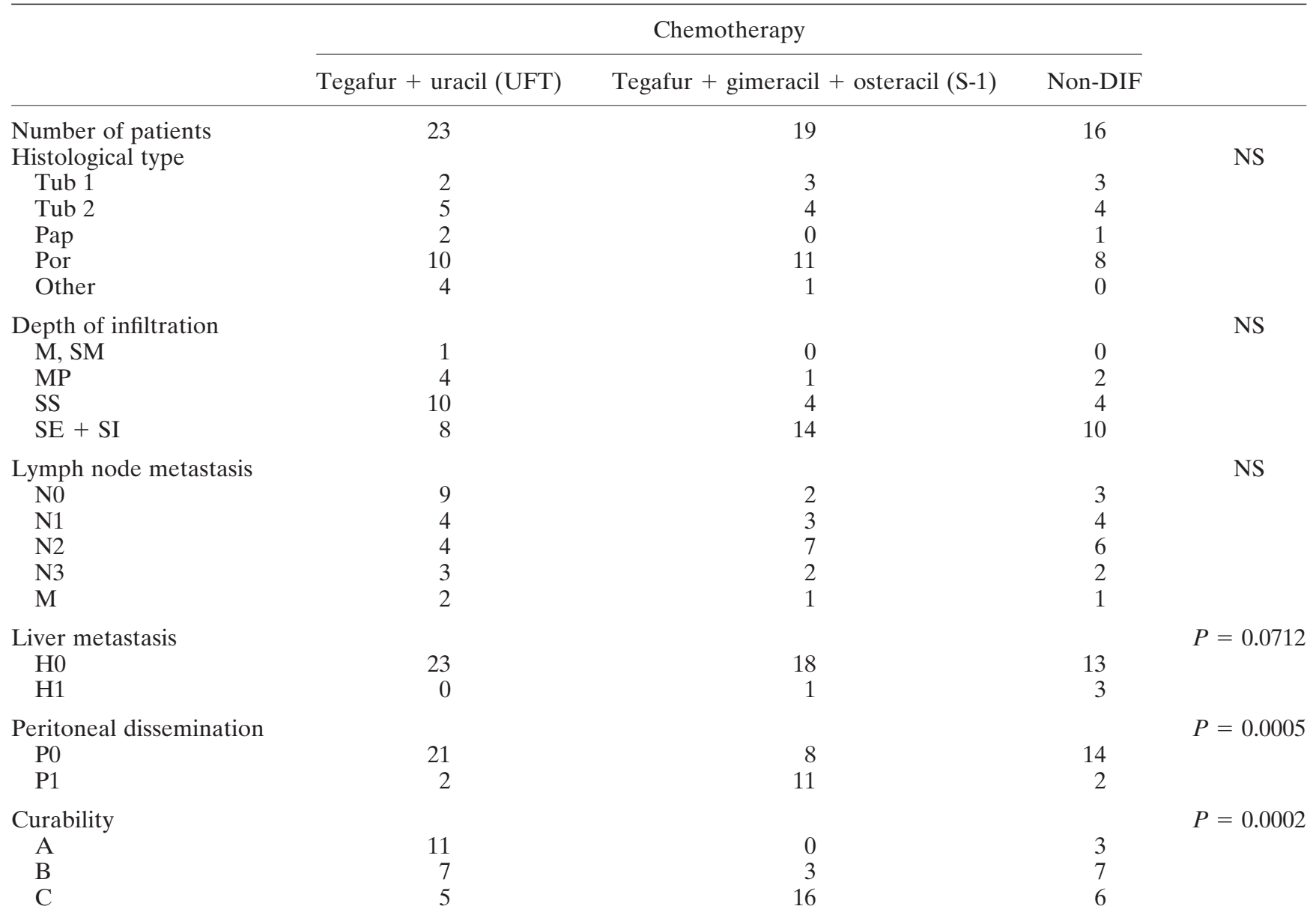

Lymph node status could not be observed in one of the patients with UFT and 4 of the patients with S-1.

Tub1, Well-differentiated tubular adenocarcinoma; tub2, moderately differentiated tubular adenocarcinoma; pap, papillary adenocarcinoma; por, poorly differentiated adenocarcinoma; M, mucosa; SM, submucosa; MP, muscularis propria; SS, subserosa; SE, serosa-exposed; SI, serosainfiltrating; DIF, dihydropyrimidine dehydrogenase inhibitory fluoropyrimidines; NS, not significant

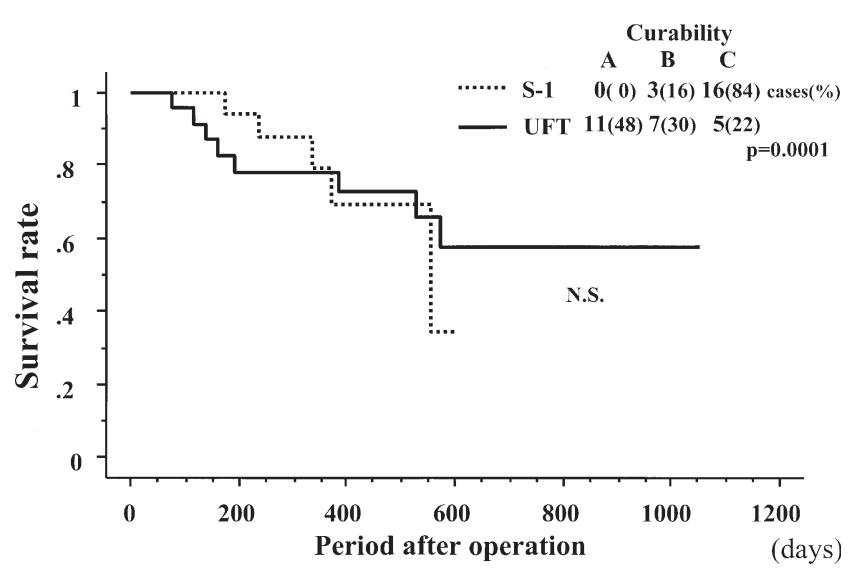

Fig. 2. Comparison of survival curves between the patients who received tegafur + uracil $(U F T)$ and the patients who received tegafur + gimeracil + osteracil $(S-1)$

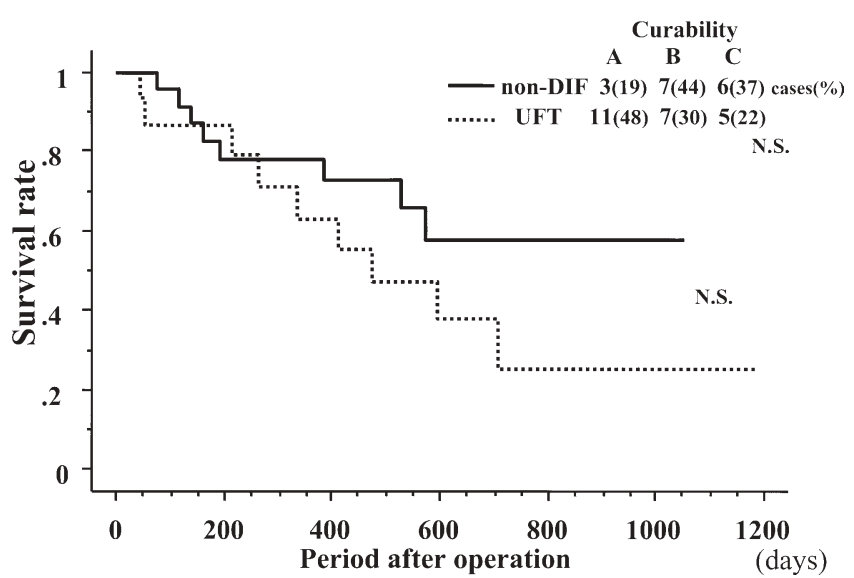

Fig. 3. Comparison of survival curves between the patients who received UFT and the patients who received other kinds of fluoropyrimidine 


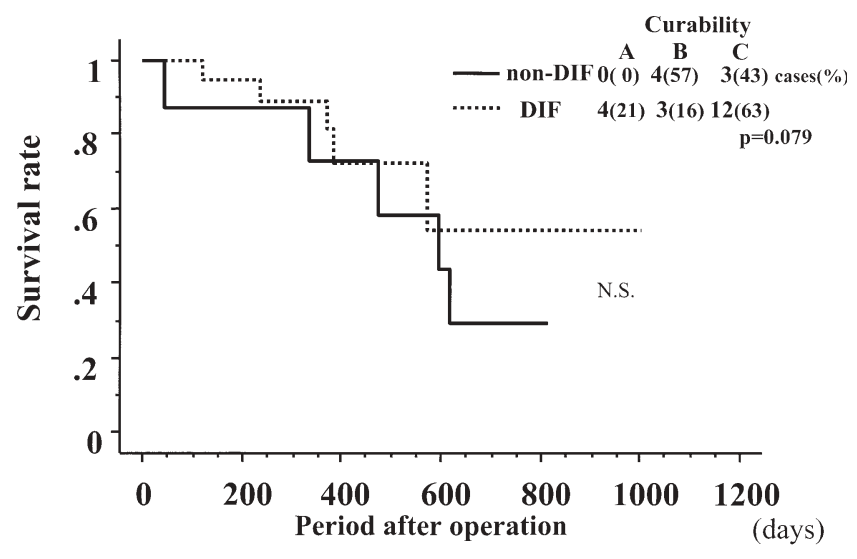

Fig. 4. Comparison of survival curves between the patients who received DIF and the patients who received other kinds of fluoropyrimidine in the group with low DPD activity

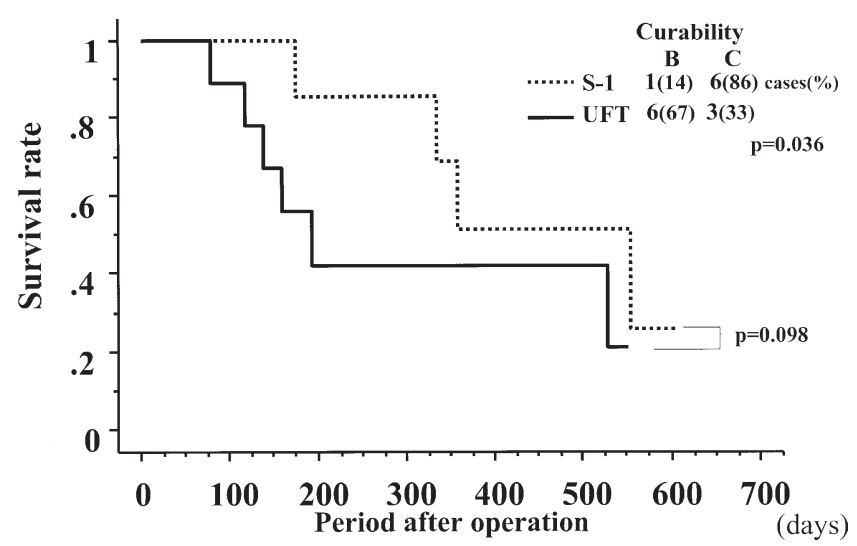

Fig. 5. Comparison of survival curves between the patients who received S-1 and the patients who received UFT in the group with high DPD expression and curability B and C

(Fig. 4). However, in the 32 patients with high DPD activity, this tendency was not observed.

Comparison of UFT and S-1. In the population with high DPD activity, prognosis tended to be better in the seven patients treated with S-1 than in the nine treated with UFT $(P=0.098)$, although those with a curative potential of $\mathrm{C}$ were significantly $(P=0.036)$ more frequent in the former than in the latter group (Fig. 5). In the population with low DPD activity, such a tendency was not observed.

\section{Response}

In 27 patients with measurable lesions, a partial response (PR) or higher response occurred in 33\% (5/15) and $\mathrm{NC}$ or higher in $53 \%(8 / 15)$ of those with low DPD activity; however, a PR or higher response occurred in $17 \%(2 / 12)$ and an NC or higher response in 33\% (4/12) of those with high DPD activity. In the patients with low
DPD activity, non-DIF induced no change (NC) in $17 \%$ $(1 / 6)$, and progressive disease (PD) in the rest. UFT induced PD in all 5 patients, while $\mathrm{S}-1$ induced a response rate of $43.8 \%$ (7/16), with NC in $25.0 \%$ (4/16). In the patients with high DPD activity, on the other hand, non-DIF $(n=3)$ and UFT $(n=3)$ induced PD in all the patients, while S-1 induced PR in 33.3\% (2/6) and NC or a higher response in $66.7 \%(4 / 6)$.

\section{Discussion}

Fluoropyrimidines have been used since the early days as chemotherapeutic agents for solid tumors, including gastric cancer. Their oral preparations in particular have been widely used in postoperative adjuvant chemotherapy conducted on an ambulatory basis [15]. Because various enzymes are involved in the in-vivo conversion of 5-fluorouracil (5-FU) to fluorodeoxyuridine monophosphate (FdUMP), which directly participates in the impairment of DNA synthesis and repair, and because drugs such as doxifluridine are converted to 5-FU by the enzyme activity in vivo, the relationship between the effect of fluoropyrimidines and the enzyme activities in the host and tumor has been arousing wide interest recently [1-6]. We focused our attention on dihydropyrimidine dehydrogenase (DPD) among these enzymes, and studied the utility of fluoropyrimidines with DPD-inhibiting activity (DIF) in cancer chemotherapy, with other fluoropyrimidines as controls.

The results showed that, though DIF were used for patients with lower curative potential when compared with other fluoropyrimidines, the survival rate in the patients treated with DIF was comparable to that in those treated with non-DIF. DIF which have been approved and are widely used in Japan are UFT and S-1. However, because gimeracil, contained in S-1, is approximately 180 times more potent than uracil in terms of DPD inhibitory activity [12], UFT and S- 1 cannot be lumped together. Actually, no difference was detected in the survival rate in those treated with UFT and those treated with non-DIF, though the former did not have lower curative potential than the latter. Accordingly, UFT, with its antitumor effect enhanced by the DPD inhibitory activity of uracil, was not more useful when compared with other fluoropyrimidines, such as leucovorin $+5 \mathrm{FU}$ (which is intended to supply 5,10-methylene tetrahydrofolate needed to form a ternary complex together with FdUMP and thymidylate synthase (TS) [46]), doxifluridine (which is considered to be effective against tumors with high thymidine phosphorylase [TP] activity $[2,3]$ ), and cisplatin +5 -FU (which is intended to provide biochemical modulation [BCM], and is widely used in Japan [16]. In the patients treated with S- 
1, although the curative potential was $\mathrm{C}$ in the majority of these patients, the survival rate was comparable to that in patients treated with non-DIF or UFT, which suggested that the strong DPD inhibitory activity of gimeracil allowed S-1 to surpass other drugs whose anticancer effect was enhanced by BCM. This was particularly conspicuous in patients with high DPD activity, which demonstrated the potent antitumor effect of S-1. The same tendency was detected in a comparison of response rate. In patients with low DPD activity, on the other hand, S-1 was not overwhelmingly more useful than other drugs. In other words, in patients with low DPD activity, the DPD inhibitory activity of uracil contained in UFT might be sufficiently useful, and drugs showing enhancement of the 5-FU effect by other methods were almost as effective as S-1. Such enhancement of effects seemed insufficient for patients with high DPD activity.

From the above results, we suggest the use of S-1, rather than UFT or other fluoropyrimidines, in patients with high DPD activity. It may also be beneficial to use S-1 not only for patients with a curative potential of C, who are the main targets of S-1 therapy at present, but also for patients with a curative potential of B. In patients with low DPD activity, S-1 also appeared useful, but when we are planning the drug treatment, the activities of other 5-FU-related enzymes, such as TS and TP, should be considered, and perhaps a sensitivity test should be added. Whatever the situation, measurement of DPD was considered useful for selecting S-1, which, in our patients, has shown favorable effects in the treatment of advanced gastric cancer.

The utility of S-1 should, of course, be verified in a randomized, controlled study. Nevertheless, to realize the concept of personalized cancer chemotherapy, we intend to continue studying the selection of anticancer drugs based not only on DPD activity but also on the activities of other enzymes as well, and we intend to study sensitivity tests for anticancer drugs, including drugs unrelated to fluoropyrimidines.

\section{References}

1. Diasio RB. Clinical implications of dihydropyrimidine dehydrogenase inhibition. Oncology (Huntingt) 1999;13:17-21.
2. Ishitsuka H, Miwa M, Takemoto K, Fukuoka K, Itoga A, Maruyama HB. Role of uridine phosphorylase for antitumor activity of 5'-deoxy-5-fluorouridine. Gann 1980;71:112-23.

3. Ohwada S, Ogawa T, Izumi M, Inoue T, Teshigawara O, Ikeya T, et al. Effects of 5'-DFUR and OK-432 on cytokines and thymidine phosphorylase in tumor tissue of gastric cancer patients. Anticancer Res 1997;17:2313-8.

4. Bertino JR, Sawicki WL, Lindquist CA, Gupta VS. Scheduledependent antitumor effects of methotrexate and 5-fluorouracil. Cancer Res 1977;37:327-8.

5. Evans RM, Laskin JD, Hakala MT. Effect of excess folates and deoxyinosine on the activity and site of action of 5-fluorouracil. Cancer Res 1981;41:3288-95.

6. Machover D, Goldschmidt E, Chollet P, Metzger G, Zittoun J, Marquet J, et al. Treatment of advanced colorectal and gastric adenocarcinomas with 5-fluorouracil and high-dose folinic acid. J Clin Oncol 1986;4:685-96.

7. Majima H. Phase I and preliminary phase II study of coadministration of uracil and FT-207 (UFT therapy). Gan To Kagaku Ryoho (Jpn J Cancer Chemother) 1980;7:1383-7.

8. Baccanari DP, Davis ST, Knick VC, Spector T. 5-Ethynyluracil (776C85): a potent modulator of the pharmacokinetics and antitumor efficacy of 5-fluorouracil. Proc Natl Acad Sci U S A 1993;90:11064-8.

9. Shirasaka T, Shimamato Y, Ohshimo H, Yamaguchi M, Kato T, Yonekura K, et al. Development of a novel form of an oral 5fluorouracil derivative (S-1) directed to the potentiation of the tumor selective cytotoxicity of 5-fluorouracil by two biochemical modulators. Anticancer Drugs 1996;7:548-57.

10. Shirasaka T, Fujita F, Fujita M, Fukushima M, Taguchi T, Fujii S. Antitumor activity and metabolism of BOF-A2, a new 5fluorouracil derivative, with human cancers xenografted in nude mice. Gan To Kagaku Ryoho (Jpn J Cancer Chemother) 1990;17: 1871-6.

11. Sasaki T. New anti-cancer drugs for gastrointestinal cancers. Gan To Kagaku Ryoho (Jpn J Cancer Chemother) 1997;24:1925-31.

12. Tatsumi K, Fukushima M, Shirasaka T, Fujii S. Inhibitory effects of pyrimidine, barbituric acid and pyridine derivatives on 5fluorouracil degradation in rat liver extracts. Jpn J Cancer Res 1987;78:748-55.

13. Mori K, Hasegawa M, Nishida M, Toma H, Fukuda M, Kubota T, et al. Expression levels of thymidine phosphorylase and dihydropyrimidine dehydrogenase in various human tumor tissues. Int $\mathbf{J}$ Oncol 2000;17:33-8.

14. Maruyama K, Sasako M, Sano T, Katai H, Kino I, Kato Y, et al. Operative procedures. In: Nishi M, Omori Y, Miwa K, editors. Japanese classification of gastric carcinoma. First English Ed. Tokyo: Kanehara; 1995. p 14-16.

15. Bateman JR, Pugh RP, Cassidy FR, Marshall GJ, Irwin LE. 5Fluorouracil given once weekly: comparison of intravenous and oral administration. Cancer 1971;28:907-13.

16. Saji S, Aiba K, Araki H, Sasaki K, Shirasaka T, Sowa M, et al. Current status of low-dose CDDP. 5-FU therapy for solid malignant tumors - nationwide questionnaire survey. Gan To Kagaku Ryoho (Jpn J Cancer Chemother) 1997;24:1892-900. 